\title{
JVIM manuscript reviewers who critiqued in the 2018 calendar year
}

The Editorial Board thanks the following individuals for their time and effort spent in reviewing and critiquing manuscripts during the 2018 calendar year.

Aceto, Helen Pennsylvania United States Acierno, Mark Arizona United States Ackerman, Mark Oregon United States Adin, Darcy Florida United States Aherne, Michael Florida United States Albasan, Hasan Minnesota United States Aleman, Monica California United States Alleman, Rick Florida United States Allenspach, Karin lowa United States Anderson, David

Andrews, Frank Louisiana United States

Antczak, Douglas New York United States

Archer, Joy United Kingdom of Great Britain and Northern Ireland Arslan, Handan Turkey

Asakawa, Midori New York United States

Attipa, Charalampos United States

Avery, Anne Colorado United States

Baan, Mieke Wisconsin United States

Bailey, Ernie Kentucky United States

Bailey, Simon Victoria Australia

Bajer, Anna Poland

Balkman, Cheryl

Bamford, Nicholas Victoria Australia

Bandt, Carsten

Baneth, Gad Israel

Bankstahl, Marion Germany

Banse, Heidi Louisiana United States

Barnes Heller, Heidi Wisconsin United States

Baron Toaldo, Marco Bologna Italy

Barrs, Vanessa New South Wales Australia

Bartges, Joeseph (Joe) Georgia United States

Barton, Ann Germany

Batchelor, Daniel United Kingdom of Great Britain and Northern Ireland

Bauquier, Jennifer Victoria Australia
Bayton, William United Kingdom of Great Britain and Northern Ireland

Beasley, Michaela Mississippi United States

Beatty, Julia New South Wales Australia

Beckmann, Katrin Switzerland

Behling-Kelly, Erica New York United States

Behrend, Ellen Alabama United States

Bell, Jerold Connecticut United States

Beltran, Elsa United Kingdom of Great Britain and Northern Ireland

Benchekroun, Ghita Île-de-France France

Berghoff, Nora Michigan United States

Bertin, Francois-Rene Queensland Australia

Bertone, Joseph California United States

Bettschart, Robert Switzerland

Bexfield, Nicholas United Kingdom of Great Britain and Northern Ireland

Bhatti, Sofie Belgium

Billen, Frédéric Belgium

Birks, Eric Delaware United States

Bisinotto, Rafael Florida United States

Bissett, Sally North Carolina United States

Blais, Marie-Claude Quebec Canada

Blois, Shauna Ontario Canada

Boari, Andrea Italy

Boileau, Melanie Oklahoma United States

Bonagura, John Ohio United States

Boothe, Dawn United States

Boretti, Felicitas Switzerland

Borgarelli, Michele Virginia United States

Borgatti, Antonella Minnesota United States

Borgeat, Kieran United Kingdom of Great Britain and Northern Ireland

Boswood, Adrian Hertfordshire United Kingdom of Great Britain and Northern Ireland

Boudreau, C. Elizabeth Texas United States

Bracha, Shay Oregon United States

Bradley, Allison Maine United States

Brainard, Benjamin Georgia United States

Brandt, Sabine Austria

Brault, Stephanie

Bayly, Warwick Washington United States

This is an open access article under the terms of the Creative Commons Attribution-NonCommercial License, which permits use, distribution and reproduction in any medium, provided the original work is properly cited and is not used for commercial purposes.

(c) 2019 The Authors. Journal of Veterinary Internal Medicine published by Wiley Periodicals, Inc. on behalf of the American College of Veterinary Internal Medicine. 
Brenner, Karen Victoria Australia

Bröjer, Johan Sweden

Brosnahan, Margaret New York United States

Brovida, Claudio Turin Italy

Brown, Cathy Georgia United States

Bryan, Jeffrey Missouri United States

Buchholz, Julia Switzerland

Buckley, Gareth Florida United States

Buczinski, Sebastien Quebec Canada

Bugbee, Andrew Georgia United States

Buhl, Rikke Denmark

Bullone, Michela Quebec Canada

Burkitt, Jamie

Burns, Teresa Ohio United States

Burton, Jenna California United States

Byers, Christopher Nebraska United States

Byrne, Barbara California United States

Byron, Julie Ohio United States

Caldwell, Marc Tennessee United States

Cardoso, Luis Portugal

Carr, Elizabeth Michigan United States

Carrera, Ines United Kingdom of Great Britain and Northern Ireland

Carslake, Harry United Kingdom of Great Britain and Northern Ireland

Carstensen, Helena Denmark

Casamian-Sorrosal, Domingo United Kingdom of Great Britain and Northern Ireland

Cassidy, Joe Ireland

Castillo, V. A. Argentina

Catalfamo, Jim New York United States

Catchpole, Brian United States

Cavalleri, Jessica Austria

Cerda-Gonzalez, Sofia Illinois United States

Cerón, José Spain

Chalermpol, Lekcharoensuk Thailand

Chamorro, Manuel Alabama United States

Chandler, Marjorie (Marge) United Kingdom of Great Britain and Northern Ireland

Chanoit, Guilaume United Kingdom of Great Britain and Northern Ireland

Charalambous, Marios Belgium

Childress, Michael Indiana United States

Chomel, Bruno California United States

Churchill, Julie Minnesota United States

Clark, Melissa Ohio United States

Clarke, Dana Pennsylvania United States

Clercx, Cecile Belgium

Coates, Joan Missouri United States

Cobb, Malcolm Leicestershire United Kingdom of Great Britain and Northern Ireland

Cohen, Todd California United States

Cohn, Leah Missouri United States

Constantino-Casas, Fernando United Kingdom of Great Britain and Northern Ireland
Cook, Audrey Texas United States

Cooke, Kirsten Florida United States

Corah, Louise United Kingdom of Great Britain and Northern Ireland

Cortese, Vic Kentucky United States

Costa, Lais Rosa Massachusetts United States

Cote, Etienne Prince Edward Island Canada

Cowgill, Larry California United States

Credille, Brent Georgia United States

Cullen, John North Carolina United States

Culshaw, Geoffrey Midlothian United Kingdom of Great Britain and Northern Ireland

Cummings, Kevin New York United States

Czuprynski, Chuck

Daminet, Sylvie Belgium

Dandrieux, Julien Switzerland

Davis, Jennifer Virginia United States

De Decker, Steven Hertfordshire United Kingdom of Great Britain and Northern Ireland

De Graaf-Roelfsema, Ellen Utrecht Netherlands

de Laat, Melody Queensland Australia

de Madron, Eric Ontario Canada

De Marco, Viviani São Paulo Brazil

De Risio, Luisa United Kingdom of Great Britain and Northern Ireland

Dear, Jonathan California United States

Decloedt, Annelies Belgium

DeClue, Amy Missouri United States

Defauw, Pieter Belgium

DeFrancesco, Teresa North Carolina United States

Dembek, Katarzyna lowa United States

Dickinson, Peter California United States

Diez de Castro, Elisa Spain

Digangi, Brian Florida United States

Diniz, Pedro Paulo California United States

Dirksen, Karen Netherlands

Dixon-Jimenez, Dixon

Dodam, John

Dodds, W. Jean California United States

Domenech, Oriol Italy

Donnell, Robert Tennessee United States

Dore, Vincent Tennessee United States

Dossin, Olivier France

Dow, Steve Colorado United States

Drögemüller, Cord Switzerland

Drost, Wm United States

Du Plessis, Wencke Saint Kitts and Nevis

Dunn, Marilyn Quebec Canada

Durando, Mary Pennsylvania United States

Durham, Andy Hampshire United Kingdom of Great Britain and Northern Ireland

Dye, Janice United States

Dyggve, Hanna Uusimaa Finland

Ekiri, Abel United Kingdom of Great Britain and Northern Ireland Elchert, Daniel Virginia United States 
Elliott, Jonathan United Kingdom of Great Britain and Northern Ireland

Epstein, Steven California United States

Estell, Krista California United States

Evans, Richard Maryland United States

Faissler, Dominik Massachusetts United States

Falk, Torkel Sweden

Feeney, Daniel Minnesota United States

Feliciano, Marcus Brazil

Fellman, Claire Massachusetts United States

Ferasin, Luca United Kingdom of Great Britain and Northern Ireland

Ferri, Filippo Novara Italy

Finno, Carrie California United States

Fischer, Andrea Bayern Germany

Fleeman, Linda Victoria Australia

Flegel, Thomas Germany

Fleming, Sherrill Mississippi United States

Fletcher, Daniel New York United States

Flethøj, Mette Denmark

Florey, Jessica United Kingdom of Great Britain and Northern Ireland

Foley, Janet United States

Fonfara, Sonja United Kingdom of Great

Britain and Northern Ireland

Forcada, Yaiza Hertfordshire United Kingdom of Great Britain and Northern Ireland

Forrest, Lisa Wisconsin United States

Foster, Derek North Carolina United States

Foster, Jonathan (JD) District of Columbia United States

Fox, Philip New York United States

Foy, Daniel Arizona United States

Fracassi, Federico Italy

Francey, Thierry Switzerland

Francisco, Amanda

Francoz, David Quebec Canada

Frank, Linda Tennessee United States

Fritz, Heather Washington United States

Fry, Michael Tennessee United States

Furr, Martin Oklahoma United States

Gal, Arnon New Zealand

Gallagher, Alexander Florida United States

Garcia, Ryan California United States

Gaschen, Frederic Louisiana United States

Gavazza, Alessandra LI Italy

Geddes, Rebecca United Kingdom of Great Britain and Northern Ireland

Geisen, Vera Germany

Gelain, Maria Italy

Gelzer, Anna Pennsylvania United States

Gerber, Vinzenz Switzerland

German, Alexander Cheshire United Kingdom of Great Britain and Northern Ireland

Geyer, Joachim Germany

Gibbons, Philippa Virginia United States
Giglio, Robson

Giguere, Steeve Georgia United States

Gilor, Chen California United States

Giuliano, Antonio United Kingdom of Great Britain and Northern Ireland

Giunti, Massimo Italy

Glaus, Tony Switzerland

Goehring, Lutz Germany

Goggs, Robert New York United States

Gold, Jennifer Washington United States

Goldberg, Katherine New York United States

Gomez, Diego Ontario Canada

Gookin, Jody North Carolina United States

Gorden, Patrick lowa United States

Gorgas, Daniela Bern Switzerland

Graham, Peter United Kingdom of Great

Britain and Northern Ireland

Granger, L. Abbigail Louisiana United States

Granger, Nicolas United Kingdom of Great Britain and Northern Ireland

Granick, Jennifer Minnesota United States

Granstrom, David Illinois United States

Grant, David Virginia United States

Griffin, Lynn Colorado United States

Grobman, Megan Missouri United States

Gronthal, Thomas United States

Grooters, Amy Louisiana United States

Grünberg, Walter Germany

Grützner, Niels Saxony-Anhalt Germany

Guglielmini, Carlo Italy

Guieu, Liz-Valery Tennessee United States

Guillaumin, Julien

Guptill-Yoran, Carolyn Indiana United States

Häggström, Jens Sweden

Hague, Devon Illinois United States

Hahn, Caroline Scotland United Kingdom of Great Britain and Northern Ireland

Haldane, Sarah Victoria Australia

Hall, Alison United Kingdom of Great Britain and Northern Ireland

Hall, Edward

Hall, Jean Oregon United States

Hamlin, Robert

Hanifeh, Mohsen

Hansen, Bernie

Harcourt-Brown, Tom Avon United Kingdom of Great Britain and Northern Ireland

Harris, Autumn Florida United States

Hart, Kelsey Georgia United States

Hartmann, Katrin Germany

Hasegawa, Daisuke Tokyo Japan

Heiene, Reidun Norway

Heilmann, Romy Saxony Germany

Heinz, Justin Texas United States 
Heller, Heidi L. Barnes United States

Heller, Meera California United States

Henke, Diana Switzerland

Herrin, Brian Kansas United States

Heseltine, Johanna Texas United States

Hess, Paul

Hess, Rebecka Pennsylvania United States

Higginbotham, Mary Lynn Kansas

United States

Hill, Tracy Georgia United States

Hines, Melissa Tennessee United States

Hoenig, Margarethe Illinois United States

Hoffman, Jessica Alabama United States

Höglund, Katja Sweden

Hollmer, Miriam Denmark

Hopper, Kate California United States

Hopster-Iversen, Charlotte Denmark

House, Amanda United States

House, John New South Wales Australia

Hughes, Kris New South Wales Australia

Hume, Kelly New York United States

Humm, Karen United Kingdom of Great Britain and Northern Ireland

Husnik, Roman Ontario Canada

Irie, Mitsuhiro Japan

Irwin, Peter Western Australia Australia

Ivens, Philip United Kingdom of Great Britain and Northern Ireland

Ives, Edward Hampshire United Kingdom of Great Britain and Northern Ireland

Jacob, Megan North Carolina United States

Jacob, Sarah Michigan United States

Jaderlund, K. Hultin United States

Jaffey, Jared Arizona United States

Jagarlamudi, Kiran Kumar Sweden

James, Fiona Ontario Canada

James, Robert Virginia United States

Jennings, Ryan Ohio United States

Jergens, Albert lowa United States

Jespersen, Thomas Denmark

Jessen, Lisbeth Denmark

Johannes, Chad lowa United States

Johnson, Amy Pennsylvania United States

Johnson, Cheri Michigan United States

Johnson, Lynelle California United States

Johnson, Susan Ohio United States

Johnston, Andrea Louisiana United States

Jokinen, Tarja Finland

Jones, Meredyth Texas United States

Jung, SeungWoo Alabama United States

Karikoski, Ninja Finland

Kass, Philip California United States

Kästner, Sabine Germany

Kathrani, Aarti United Kingdom of

Great Britain and Northern Ireland
Katz, Lisa Ireland

Keene, Bruce North Carolina United States

Kempf, Jennifer Germany

Kemppainen, Robert Alabama United States

Kent, Andrew United Kingdom of Great Britain and Northern Ireland

Kent, Michael California United States

Kerl, Marie Missouri United States

Kidd, Linda California United States

Kieler, Ida Denmark

Kietzmann, Manfred Germany

Kipar, Anja ZH Switzerland

Kisielewicz, Caroline United Kingdom of

Great Britain and Northern Ireland

Kittleson, Mark California United States

Klahn, Shawna Virginia United States

Knapp, Deborah Indiana United States

Knowler, Susan Surrey United Kingdom of Great Britain and Northern Ireland

Knowles, Edd

Knych, Heather California United States

Kogan, Lori United States

Kogika, Marcia Sao Paulo Brazil

Kohn, Barbara Germany

Kol, Amir California United States

Kooistra, Hans Netherlands

Kook, Peter Switzerland

Kraus, Marc Pennsylvania United States

Kromhout, Kaatje Belgium

Kruger, John Michigan United States

Kruitwagen, Hedwig Netherlands

Kuca, Thibaud Switzerland

KuKanich, Butch Kansas United States

KuKanich, Kate Kansas United States

Kuleš, Josipa Croatia

Labato, Mary Anna Massachusetts United States

Lakritz, Jeff Ohio United States

Langhorn, Rebecca Denmark

Larsen, Jennifer California United States

Larson, Martha United States

Lathan, Patty Mississippi United States

Lavergne, Sidonie Illinois United States

Lawrence, Yuri Texas United States

Le Boedec, Kevin France

LeBlanc, Amy Maryland United States

Leclere, Mathilde Quebec Canada

Lee, Alice Che Yu Florida United States

Lee, Seunggon Korea, Republic of

Lee, Ya-Jane Taiwan

Leeb, Tosso Switzerland

Lee-Fowler, Tekla Alabama United States

Leguillette, Renaud Alberta Canada

Leisewitz, Andrew Gauteng South Africa

Lennon, Elizabeth Tennessee United States 


\section{American College of
Veterinary Internal Medicine}

Levine, Jonathan Texas United States

Li, Ronald California United States

Lidbury, Jonathan Texas United States

Liebel, Francois United Kingdom of Great Britain and Northern Ireland

Lindåse, Sanna Sweden

Little, Susan Ontario Canada

Ljungvall, Ingrid Sweden

Lobetti, Remo South Africa

Locatelli, Chiara Italy

Londono, Leo Florida United States

Looper, Jayme Louisiana United States

López-Alvarez, Jordi Spain

Lorenz, Ingrid Germany

Lottati, Maya California United States

Loureiro, Joao Surrey United Kingdom of Great Britain and Northern Ireland

Luby, Christopher Saskatchewan Canada

Lucy, John New York United States

Luis Fuentes, Virginia United Kingdom of Great Britain and Northern Ireland

Luis-Lima, Sergio Spain

Lulich, Jody Minnesota United States

Lundberg, Alycen Illinois United States

Luther, Linda New Hampshire United States

Luttle, Susan Oklahoma United States

Lutz, Thomas Switzerland

Lux, Cassie Tennessee United States

Lynch, Alex

MacDonald, Kristin California United States

Macfarlane, Laura United Kingdom of Great Britain and Northern Ireland

MacKay, Robert Florida United States

Maddox, Thomas

Maeda, Shingo Japan

Mair, Tim Kent United Kingdom of Great Britain and Northern Ireland

Majoy, Sean Massachusetts United States

Malalana, Fernando

Mallicote, Martha

Mama, Khursheed Colorado United States

Mandell, Deborah Pennsylvania United States

Mann, Sabine New York United States

Mansfield, Caroline Victoria Australia

Marks, Stanley California United States

Marsilio, Sina Texas United States

Martinez-Pereira, Yolanda Midlothian United Kingdom of Great Britain and Northern Ireland

Martin-Jimenez, Tomas Tennessee United States

Mastormauro, Michael North Carolina United States

Matiasek, Kaspar Bavaria Germany

Mattoon, John Washington United States

Matz, Brad

Mauler, Daniela Missouri United States

Mayer, Monique Saskatchewan Canada

Mayhew, Joe New Zealand
Mazan, Melissa Massachusetts United States

McAnulty, Jonathan

McCleary-Wheeler, Angela New York United States

McClure, J. Trenton Prince Edward Island Canada

McDonnell, John Maryland United States

McFarlane, Dianne Oklahoma United States

McGuirk, Sheila

McKenzie, Brennan

McMichael, Maureen Illinois United States

Menciotti, Giulio Virginia United States

Mendes, Luiz Sao Paulo Brazil

Menzies-Gow, Nicola Herts United Kingdom of Great Britain and Northern Ireland

Metzger, Julia Germany

Meyerhoff, Nina Lower Saxony Germany

Miller, Andrew New York United States

Miller, Donald New York United States

Minamoto, Yasushi Texas United States

Mitchell, Katharyn ZH Switzerland

Mittel, Linda New York United States

Mochel, Jonathan lowa United States

Moon, Rachel Alabama United States

Moore, George Indiana United States

Moore, Sarah Ohio United States

Mor, Kumar Minnesota United States

Morán, Gabriel

Moreno, Paloma Victoria Australia

Morgan, Ruth United States

Morresey, Peter Kentucky United States

Mudge, Margaret Ohio United States

Mulon, Pierre-Yves Tennessee United States

Muñana, Karen North Carolina United States

Murtaugh, Bob United States

Mutsaers, Anthony Ontario Canada

Nabity, Mary Texas United States

Navas de Solís, Cristobal Texas United States

Naylor, Jonathan Saskatchewan Canada

Naylor, Rosie Hertfordshire United Kingdom of Great Britain and Northern Ireland

Neel, Jennifer North Carolina United States

Nelli, Rahul Michigan United States

Nessler, Jasmin Germany

Niehaus, Andrew Ohio United States

Nolan, Michael North Carolina United States

Nolen-Walston, Rose Pennsylvania United States

Norton, Elaine Minnesota United States

Nout, Yvette California United States

Novellas, Rosa

Nykamp, Stephanie Ontario Canada

Oberhaus, Erin Louisiana United States

O'Brien, Dennis Missouri United States

Ohno, Koichi Japan

Olby, Natasha North Carolina United States 
Olin, Shelly Tennessee United States

Oliveira, Cintia Illinois United States

Oliveira, Pedro Italy

Ollivett, Terri Wisconsin United States

Ollivett, Theresa Ontario Canada

Olsen, Lisbeth Denmark

O'Neill, Emma Dublin Ireland

O'Neill, Kelly Massachusetts United States

Orton, Chris Colorado United States

Osterstock, Jason Michigan United States

Oyama, Mark Pennsylvania United States

Paciello, Orlando

Packer, Rowena United Kingdom of Great Britain and Northern Ireland

Paepe, Dominique Belgium

Pagitz, Maximilian Austria

Pakozdy, Akos Austria

Palm, Carrie California United States

Pan, Xuan Wisconsin United States

Panciera, David Virginia United States

Papich, Mark North Carolina United States

Pardon, Bart Belgium

Pariaut, Romain New York United States

Parker, Valerie Ohio United States

Patterson Kane, Janet Colorado United States

Patterson, Edward (Ned) Minnesota United States

Pearl, David Ontario Canada

Peek, Simon Wisconsin United States

Penning, Louis Utrecht Netherlands

Peremans, Kathelijne

Pesavento, Patricia California United States

Peterson, Mark New York United States

Phillips, Heidi Illinois United States

Pieper, Jason Illinois United States

Piercy, Richard United Kingdom of Great Britain and Northern Ireland

Pirie, Robert (Scott) Scotland United Kingdom of Great Britain and Northern Ireland

Platt, Simon Georgia United States

Pluhar, G. Elizabeth Minnesota United States

Podell, Michael Illinois United States

Polzin, David Minnesota United States

Pomrantz, Jill California United States

Poncelet, Luc Belgium

Poole, David

Porciello, Francesco Italy

Poulsen, Keith Wisconsin United States

Prado, Maria Tennessee United States

Pressler, Barrak California United States

Prutton, James Hampshire United Kingdom of Great Britain and Northern Ireland

Pypendop, Bruno California United States

Quimby, Jessica Ohio United States

Raj, Karthik Pennsylvania United States

Ramirez, Alejandro lowa United States
Ramos, Daniela

Ramsey, Ian United Kingdom of Great Britain and Northern Ireland

Rankin, Shelley Pennsylvania United States

Reagan, Krystle California United States

Reed, Stephen Kentucky United States

Reetz, Jennifer Pennsylvania United States

Refsal, Kent Michigan United States

Reineke, Erica Pennsylvania United States

Reinero, Carol Missouri United States

Rendle, David Hampshire United Kingdom of Great Britain and Northern Ireland

Renshaw, Randall New York United States

Rentko, Virginia United States

Rhinehart, Jaylyn Ohio United States

Richard, Eric France

Riedesel, Elizabeth lowa United States

Robben, Joris Utrecht Netherlands

Roberts, Veronica North Somerset United Kingdom of Great Britain and Northern Ireland

Robinson, Mary

Rondeau, Mark Pennsylvania United States

Rosenthal, Melisa Massachusetts United States

Rossmeisl, John Virginia United States

Roussel, Allen Texas United States

Roy, Marie-France Alberta Canada

Royal, Kenneth North Carolina United States

Rozanski, Elizabeth Massachusetts United States

Ruaux, Craig New Zealand

Rudinsky, Adam Ohio United States

Rush, John Massachusetts United States

Rütgen, Barbara Kragujevac Austria

Rutter, Chrissie

Rylander, Helena Wisconsin United States

Sacco, James lowa United States

Saelinger, Carley California United States

Saker, Korinn

Salavati Schmitz, Silke Germany

Sangster, Jodi Virginia United States

Santilli, Roberto Varese Italy

Sargeant, Jan Ontario Canada

Sargent, Julia United Kingdom of Great Britain and Northern Ireland

Savvas, Ioannis Greece

Scansen, Brian Colorado United States

Schenck, Patricia Michigan United States

Scherk, Margie

Schermerhorn, Thomas Kansas United States

Schleis, Stephanie Alabama United States

Schneider, Matthias Germany

Schoeman, Johan Gauteng South Africa

Schoenfeld, Regina North Carolina United States

Schott II, Harold Michigan United States

Schott, Hal Michigan United States

Schroeder, Eric Ohio United States 
Schrope, Donald New Jersey United States

Scorza, Andrea Colorado United States

Scott, Katherine United States

Scott-Moncrieff, Catharine Indiana United States

Secombe, Cristy Australian Capital Territory Australia

Segev, Gilad Israel

Sellon, Rance Washington United States

Selting, Kim Illinois United States

Serrano, Goncalo Belgium

Seth, Mayank Essex United Kingdom of Great Britain and Northern Ireland

Sharkey, Lesley Massachusetts United States

Shaw, Thomas United Kingdom of Great Britain and Northern Ireland

Shelton, G. Diane California United States

Shipov, Anna Israel

Shores, Andy Mississippi United States

Shropshire, Sarah Colorado United States

Silva, Joel United Kingdom of Great Britain and Northern Ireland

Silverstein, Deborah Pennsylvania United States

Simpson, Missy Colorado United States

Slack, JoAnn United States

Sleeper, Margaret (Meg) Florida United States

Slovak, Jennifer Washington United States

Slovis, Nathan Kentucky United States

Smee, Nicole Kansas United States

Smith, Joe lowa United States

Smith, Sarah United Kingdom of Great Britain and Northern Ireland

Smith, Stephanie Illinois United States

Soboll Hussey, Gisela Michigan United States

Sojka Kritchevsky, Janice Indiana United States

Southwood, Louise

Spada, Eva Italy

Spalla, Ilaria United Kingdom of Great Britain and Northern Ireland

Specht, Andrew Florida United States

Spee, Bart Netherlands

Stauthammer, Christopher Minnesota United States

Steffen, Frank Switzerland

Steinbach, Sarah United States

Stepaniuk, Kevin Washington United States

Stephenson, Hannah Lancashire United Kingdom of Great Britain and Northern Ireland

Stepien, Rebecca Wisconsin United States

Stern, Joshua California United States

Strand, Elizabeth Tennessee United States

Straub, Mary California United States

Stull, Jason Ohio United States

Suchodolski, Jan Texas United States

Sullivan, Stacey Australia

Suter, Steven North Carolina United States

Suzuki, Ryohei Tokyo Japan

Sweeney, Corinne Pennsylvania United States

Swift, Simon Florida United States

Sykes, Benjamin (Ben) Queensland Australia
Syme, Harriet Herts United Kingdom of Great Britain and Northern Ireland

Szabo, David California United States

Szacawa, Ewelina lubelskie Poland

Szatmári, Viktor Utrecht Netherlands

Szivek, Anna Florida United States

Taboada, Joseph Louisiana United States

Tadros, Elizabeth Michigan United States

Tadros, Lisa Michigan United States

Takano, Hiroshi Kanagawa Japan

Tamborini, Alice Ireland

Tamzali, Youssef France

Tasker, Séverine United Kingdom of Great Britain and Northern Ireland

Tefft, Karen

Teske, Erik Netherlands

Thamm, Douglas Colorado United States

Thawley, Vince Pennsylvania United States

Thieman Mankin, Kelley Texas United States

Thomas, William Tennessee United States

Thomovsky, Elizabeth Indiana United States

Thrall, Mary Anna

Tidholm, Anna Sweden

Timsit, Edouard Alberta Canada

Tjostheim, Sonja Wisconsin United States

Tolbert, Katie Texas United States

Toribio, Ramiro Ohio United States

Torres-Henderson, Camille Colorado United States

Totton, Sarah

Tou, Sandra North Carolina United States

Trefz, Florian Germany

Trepanier, Lauren Wisconsin United States

Trott, Darren South Australia Australia

Tuohy, Joanne Colorado United States

Uriarte, Ane Massachusetts United States

Vaden, Shelly North Carolina United States

Valberg, Stephanie Michigan United States

Van de Walle, Gerlinde New York United States

van der Meer, Frank Alberta Canada

van Erck, Emmanuelle Belgium

van Galen, Gaby Denmark

van Loon, Gunther Belgium

Van Wettere, Arnaud Utah United States

Vandevelde, Marc Switzerland

Visser, Lance California United States

Vitt, Jordan Illinois United States

von Euler, Henrik

Votion, Dominique Liège Belgium

Wakayama, Justin Hawaii United States

Wakshlag, Joseph (Joe) New York United States

Waldridge, Bryan Kentucky United States

Walker, Julie Wisconsin United States

Wallace, Mandy 
Waller, Andrew United Kingdom of Great Britain and Northern Ireland Walton, Raquel Pennsylvania United States

Wang, Chong lowa United States

Wang-Leandro, Adriano Lower Saxony Germany

Ward, Jessica lowa United States

Wardrop, Jane Washington United States

Warry, Emma Ohio United States

Washabau, Robert Minnesota United States

Watson, Penny United Kingdom of Great Britain and Northern Ireland

Webb, Craig Colorado United States

Webster, Cynthia Massachusetts United States

Weese, J. (Scott) Ontario Canada

Weishaar, Kristen Colorado United States

Weltman, Joel New York United States

Wennogle, Sara Colorado United States

Werners, Arno St. George Grenada
Wessmann, Annette United Kingdom of Great Britain and Northern Ireland

Westropp, Jodi California United States

Whittemore, Jacqueline Tennessee United States

Wilkins, Pamela Illinois United States

Wilkinson, Tom Washington United States

Wilson, Heather Texas United States

Winston, Jenessa North Carolina United States

Wisner, Erik California United States

Wittek, Thomas Austria

Wong, David lowa United States

Wood, Michael Wisconsin United States

Wrzosek, Marcin Poland

Yamasaki, Masahiro Iwate Japan

Yerramilli, Murthy Maine United States

Yoo, Seung Washington United States

Yoon, Junghee Korea, Republic of 